\title{
The origin and maintenance of montane diversity: integrating evolutionary and ecological processes
}

\author{
Catherine H. Graham, Ana C. Carnaval, Carlos Daniel Cadena, Kelly R. Zamudio, Trina E. Roberts, \\ Juan Luis Parra, Christy M. McCain, Rauri C. K. Bowie, Craig Moritz, Stephen B. Baines, \\ Christopher J. Schneider, Jeremy VanDerWal, Carsten Rahbek, Kenneth H. Kozak and \\ Nathan J. Sanders
}

C. H. Graham (catherine.graham@stonybrook.edu), J. L. Parra and S. B. Baines, Dept of Ecology and Evolution, Stony Brook Univ., Stony Brook, NY 11794-5245, USA. JLP also at: Inst. de Biologia, Facultad de Ciencias Exactas y Naturales, Univ. de Antioquia, Medellin, Colombia. - A. C. Carnaval, Dept of Biology, City College and City Univ. of New York, New York, NY 10031, USA. - C. D. Cadena, Laboratorio de Biología Evolutiva de Vertebrados, Depto de Ciencias Biológicas, Univ. de los Andes, Apartado 4976, Bogotá, Colombia. - K. R. Zamudio, Cornell Univ., Dept of Ecology and Evolutionary Biology, E209 Corson Hall, Ithaca, NY 14853-2701, USA. - T. E. Roberts, National Evolutionary Synthesis Center, 2024 W. Main Street, Suite A200, Durham, NC 27705-4667, USA, and Univ. of Iowa, Museum of Natural History, Iowa City, IA 52242, USA. - C. M. McCain, Dept of Ecology and Evolutionary Biology and CU Museum of Natural History, Univ. of Colorado, Boulder, CO 80309, USA. - R. C. K. Bowie and C. Moritz, Museum of Vertebrate Zoology and Dept of Integrative Biology, Univ. of California-Berkeley, Berkeley, CA 94720-3160, USA. - C. J. Schneider, Dept of Biology, Boston Univ., Boston, MA 02215, USA. -J. VanDerWal, Centre for Tropical Biology and Climate Change Research, School of Marine and Tropical Biology, James Cook Univ., Townsville, QLD 4811, Australia. - C. Rabbek and N. J. Sanders, Center for Macroecology, Evolution, and Climate, Dept of Biology, Univ. of Copenhagen, Universitetsparken 15, DK-2100 Copenhagen O, Denmark. NJS also at: Dept of Ecology and Evolutionary Biology, Univ. of Tennessee, Knoxville, TN 37996, USA. - K. H. Kozak, Bell Museum of Natural History and Dept of Fisheries, Wildlife, and Conservation Biology, Univ. of Minnesota, St Paul, MN 55108, USA.

\begin{abstract}
Determining how ecological and evolutionary processes produce spatial variation in local species richness remains an unresolved challenge. Using mountains as a model system, we outline an integrative research approach to evaluate the influence of ecological and evolutionary mechanisms on the generation and maintenance of patterns of species richness along and among elevational gradients. Biodiversity scientists interested in patterns of species richness typically start by documenting patterns of species richness at regional and local scales, and based on their knowledge of the taxon, and the environmental and historical characteristics of a mountain region, they then ask whether diversity-environment relationships, if they exist, are explained mostly by ecological or evolutionary hypotheses. The final step, and perhaps most challenging one, is to tease apart the relative influence of ecological and evolutionary mechanisms. We propose that elucidating the relative influence of ecological and evolutionary mechanisms can be achieved by taking advantage of the replicated settings afforded by mountains, combined with targeted experiments along elevational gradients. This approach will not only identify potential mechanisms that drive patterns of species richness, but also allow scientists to generate more robust hypotheses about which factors generate and maintain local diversity.
\end{abstract}

Spatial variation in diversity results from the interplay of ecological and evolutionary mechanisms acting over contemporary and historical time frames (MacArthur 1972, Graham et al. 2006, Ricklefs 2006, Jetz et al. 2012). Numerous attempts have been made to evaluate the relative roles of ecological and evolutionary factors that operate at diverse spatial and temporal scales in shaping patterns of diversity (Terborgh and Faaborg 1980, Rahbek and Graves 2001, Hawkins et al. 2005, Stevens 2006, Rabosky 2009, Kozak and Wiens 2010). However, a general framework that guides the integration of different mechanisms to understand the interplay of ecology and evolution on diversity patterns is only just emerging.
Generally speaking, this emerging framework consists of conceptual and methodological advances targeted at understanding patterns of diversity that fall into three general categories. First, attempts targeted at understanding spatial variation in species richness evaluate both ecological environmental correlates (e.g. energy) and evolutionary mechanisms (e.g. time for speciation; Li et al. 2009, Kozak and Wiens 2012). Second, evolutionary approaches now explicitly acknowledge that ecological interactions, such as interspecific interactions and niche partitioning, might place upper limits on the number of species that can occur in a given area. These local-scale phenomena are now considered in attempts to evaluate diversity patterns and 
speciation rates (Cavender-Bares et al. 2004, Rabosky 2009, Rabosky and Glor 2010, Stevens 2011, Machac et al. 2013). Third, studies of local assemblages increasingly consider the influence of processes acting at broad spatial scales on local diversity (MacArthur and Levins 1967, Ricklefs 2004, Harrison and Grace 2007, Harrison and Cornell 2008). A common theme in all of these approaches is that ecological and evolutionary mechanisms generating local diversity patterns can act at multiple spatial and temporal scales, making it challenging to determine the relative importance of different mechanisms (Wiens 1989, Cavender-Bares et al. 2009).

Our goal is to build on the current integrative research described above and explore an approach for identifying the underlying ecological and evolutionary mechanisms that influence pattern of species richness. While it may not yet be feasible to determine definitively the causal mechanisms for any given diversity gradient - one of the holy grails of ecology and evolution - our approach provides a series of steps to begin to tease apart the relative importance of various drivers of species richness. We do not attempt to review the numerous ecological and evolutionary drivers of richness, as comprehensive reviews already exist (Willig et al. 2003, Currie et al. 2004, Mittelbach et al. 2007). We focus on mountain systems for a variety of reasons: they differ in age, size, historical stability, climate regimes and topographic complexity making them excellent natural laboratories to determine how environmental, geographic and biotic factors interact to promote diversification and maintenance of mountain flora and fauna. Importantly, many species inhabiting montane diversity hotspots are endemic to relatively restricted and fragmented high-elevation habitats (Graves 1985, Rahbek 1997) and may be especially subject to the potential impact of climate change (La Sorte and Jetz 2010, McCain and Colwell 2011); though it may also be possible for some of these species to track their thermal niche (Loarie et al. 2009, Bertrand et al. 2011). Consequently, understanding the causes of elevational diversity gradients is critical to predicting how patterns of species richness may change in response to projected global changes in climate. Nonetheless, the general framework developed herein for mountains could be applied to other regional gradients such as withinbiome or bathymetric gradients.

\section{A naturally replicated natural experiment: mountains as models}

Elevational gradients represent an appropriate system for evaluating whether general relationships exist among richness patterns, environmental variables, and the ecologi$\mathrm{cal}$ and evolutionary processes that determine how many species co-occur in a given area (Rahbek 1995, Körner 2000). A variety of richness patterns occur on mountains: the most prevalent are low- and mid-elevation peaks in richness (Rahbek 1995, 2005, McCain and Grytnes 2010, Werenkraut and Ruggiero 2011), although some instances of increasing richness at high elevations (Sanders et al. 2003) or no change in richness with elevation (Fierer et al. 2011) have been documented. There are hundreds of montane gradients distributed across the globe which differ in age, size, connectivity, persistence of habitat through time (i.e. stability), topographic complexity, climatic regimes and other environmental attributes, making comparative studies possible. Thoughtful selection of montane gradients for study, focused on specific hypotheses or questions, can illuminate some of the mechanisms driving diversity patterns (Kattan and Franco 2004, Körner 2007). For instance, because particular elevational gradients are nested within single biogeographic regions, it may be possible to minimize the effects of large-scale environmental variation (e.g. climatic seasonality), which typically complicates studies along latitudinal gradients (Körner 2000, Sundqvist et al. 2013). In addition, and in sharp contrast with latitudinal gradients, elevational gradients often span many climatic zones across a short distance and thus provide higher variance in climate within dispersal distances of many species than do latitudinal gradients. While different mechanisms might be at play along latitudinal and elevational gradients, making it potentially problematic to generalize mechanisms identified on elevational gradients to latitudinal gradients, the enhanced climatic variation over short spatial scales makes mountains an ideal study system to examine at least some of the mechanisms underlying spatial variation in biodiversity. Another benefit of mountain systems compared to latitudinal gradients is that montane systems often have a more limited species pool from which locally coexisting species are drawn whereas latitudinal studies often include sites from distinct species pools and unique evolutionary histories, making it more difficult to disentangle ecological and evolutionary factors that can affect patterns of species richness. Finally, though the number of experiments and observations along latitudinal gradients is increasing (Schemske et al. 2009), it is often much more tractable to conduct experiments and make repeated observations along elevational gradients than latitudinal gradients.

\section{An approach for combining ecological and evolutionary mechanisms}

It was long thought that species richness simply declined linearly with elevation (MacArthur 1972), but that is often not the case; a variety of richness patterns occur on mountains and these patterns can be generated by a range of different mechanisms (Rahbek 2005). For simplicity, we begin to explore these mechanisms by considering patterns of species richness along a single elevational gradient, though elevation is likely not the key environmental gradient influencing richness (i.e. no organisms except humans walk around with altimeters and move up or down in elevation because of what the altimeter says; Körner 2007), but is likely a proxy for spatial variation in the environment (elaborated on below). To develop our approach, we first define the terms as we apply them here, because many have been used in a variety of ways in the literature. Second, we explore mechanisms that influence observed patterns of local species richness (i.e. the number of species in a local community) across an elevational gradient. Third, we describe a research approach to explore how different eco-evolutionary mechanisms interact to produce current patterns of species richness across gradients. Fourth, we identify research challenges 
associated with applying our approach and offer perspectives on how our framework can provide new research directions.

\section{Terminology: scale, region and species pools}

Observed patterns of species richness across a gradient reflect mechanisms acting at different spatial and temporal scales. Here we define two spatial scales, regional and local, and consider mechanisms that generally act over long time frames (thousands to millions of years) and shorter time frames (tens to hundreds of years). We use 'montane region' to mean an entire mountain range with a unique history that is distinct from that of neighboring, but biogeographically separate ranges. Mechanisms that generate and maintain species richness within regions, e.g. speciation, extinction and colonization from outside the region, generally act across 'long time' frames. We use 'local scale' to refer the scale over which species can interact, either directly or indirectly, with outer species co-occurring with them in space and time. Depending on the organisms local can vary in size by orders of magnitude. At this scale, evolutionary processes such as speciation are unlikely to occur (Losos and Schluter 2000, Kisel and Barraclough 2010). While some evidence suggests that speciation, in particular, can occur at small spatial scales and over short time periods, full consideration of contemporary evolution is beyond the scope of this paper (Hendry and Kinnison 1999).

A species pool refers to the number and identity of species in a given region and is often used to evaluate whether patterns of species richness or composition in a local assemblage, or sub-region within this broader region, differ more than that expected by chance when compared to the species in the broad region (Gotelli 2000). There is no single way to define the region from which the species pool is taken; instead species pools can be defined considering different geographic extents (subregions within a broad region) or subsets of environmental conditions and as such can be used to identify specific mechanisms that structure patterns of species richness (Algar et al. 2011, Lessard et al. 2012, Carstensen et al. 2013).

\section{Mechanisms influencing gradients in species richness}

Rates of speciation, extinction or colonization vary with environmental conditions along elevational gradients, causing the number of species that could potentially occur at any point along the gradient to vary (Fig. 1). Patterns of species richness along elevational gradients may partly result from the rate of lineage diversification (the balance of speciation and extinction) and the time that lineages have persisted in a portion of the gradient. Parts of the gradient where lineages diversify quickly, or where lineages have been present for longer, are expected to be more diverse (Wiens et al. 2007). Colonization from outside the region can also influence patterns of species richness, because if colonizing species are adapted to conditions at a particular part of the gradient, then they may be more likely to establish and potentially diversify under these conditions (Donoghue 2008, Drummond 2008). Yet, diversification rate, persistence time, and colonization need not co-vary across the gradient. In fact, combinations of these processes can result in similar patterns of species richness across a gradient (Fig. 1). For instance, a uniform pattern of richness along a gradient (Fig. 1g) could be the result of a uniform diversification rate and lineage persistence time (Fig. 1e) or opposite patterns of diversification rate and lineage persistence time (Fig. 1d). In addition, it might also be the case that local-scale interactions scale up to influence diversification dynamics and ultimately the regional species pool, though this is only beginning to be explored (Rabosky 2009, Rabosky and Glor 2010). Interactions among species in sympatry can result in character displacement (Schluter and McPhail 1992, Adams and Rohlf 2000, Davies et al. 2007) which could influence the availability of empty niches or ecological opportunity in a given region (Rabosky 2009, Rabosky and Glor 2010). We refer to the number and identity of species that could occur in a given elevational band across the entire mountain region given these mechanisms that act over relatively long temporal scales (see terminology above) as the potential pool, which is usually a subset of those species that can occur within the entire mountain region (regional pool). While determining the number of potentially colonizing species accurately will often be challenging, it serves as a conceptual and heuristic tool for our framework and likely can be approximated based on species regional distribution patterns and the match between species and the local environment (see below). For instance, given knowledge of the thermal tolerances of all ant species in the southern Appalachia and the temperatures at local sites an approximate potential pool could be estimated (Diamond et al. 2012).

Local gradients will emerge from an additional set of processes that may further modify the potential pattern of species richness generated by speciation, extinction and regional colonization described above. For example, certain species in a potential pool might not occur in local assemblages at particular elevations because of competing species or lack of mutualists (Cavieres et al. 2014). Such biotic interactions limit the number of species from the potential pool that can persist in local communities along the gradient, causing discordance between the potential and observed local patterns of species richness (Fig. 1, panel II and III). Overlap in the richness pattern of the potential species pool to observed richness (Fig. 1h) might indicate that historical diversification and colonization processes are largely responsible for the observed patterns of species richness and that local biotic interactions have, at best, only a weak effect (MacArthur 1972, Terborgh and Faaborg 1980, Wiens et al. 2007). In contrast, discordance between potential richness patterns and observed local patterns (Fig. 1 $\mathrm{i}-\mathrm{m}$ ) is expected if local biotic interactions have influenced the assembly of local assemblages from the potential pool (Terborgh and Faaborg 1980, Sanders et al. 2003, Rabosky 2009, Rabosky and Glor 2010, Cavieres et al. 2014). In the latter cases, constraints due to biotic interactions might reduce or even reverse the pattern expected based on the potential species pool along the gradient. When species richness in the potential pool does not vary with elevation (Fig. 1g), biotic interactions that exclude species in different elevational bands may influence observed richness patterns (Fig. $1 \mathrm{k}-\mathrm{m}$ ). 


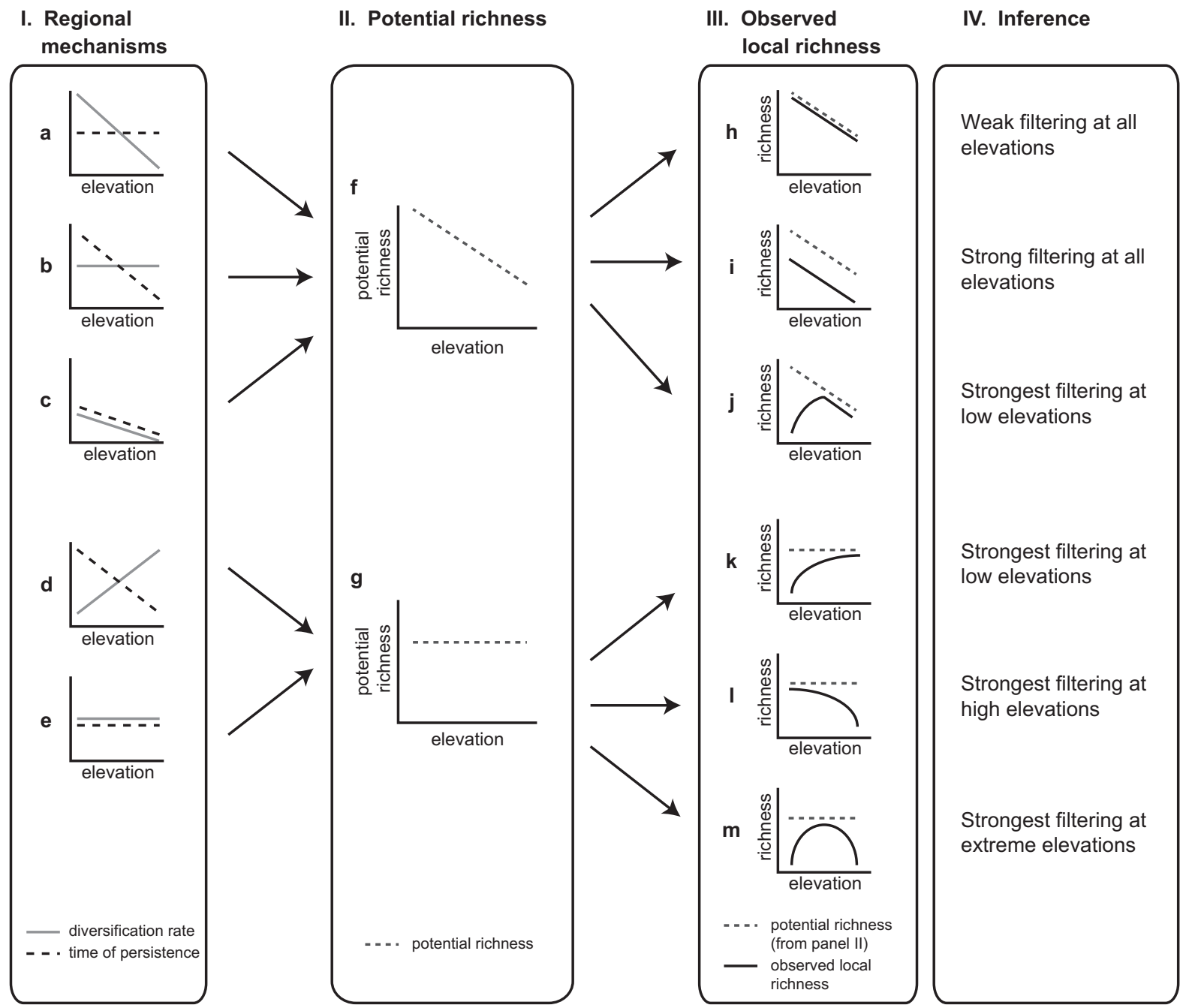

Figure 1. Conceptual framework integrating evolutionary and ecological approaches to studying mechanisms contributing to local patterns of diversity along elevational gradients. Diversification rate and time of lineage persistence (panel I) combine to generate potential patterns of diversity along the gradient (panel II). Colonization can also influence potential patterns of diversity, but for visual clarity it is not shown. Ecological filtering acts to produce the observed local richness pattern (panel III) which can take various forms.

The combinations shown in Fig. 1 do not represent all possible interactions between evolutionary and ecological mechanisms that shape empirical richness patterns. However, these examples underscore the complexity of possible interactions, the multiple paths by which different mechanisms yield similar outcomes, and the importance of evaluating how historical and contemporary mechanisms interact when inferring the causes of observed diversity patterns. While this heuristic framework may be conceptually useful, we realize it can be difficult to implement. Below we outline a series of steps that can facilitate integrative analyses of patterns of montane richness patterns, which can then be compared across other richness gradients.

\section{Research steps for evaluating mechanisms influencing montane diversity}

Elevation per se does not affect the patterns of species richness along elevational gradients (Rahbek 1995, Körner
2000). Elevational gradients represent many different environmental gradients and historical factors (e.g. productivity, temperature, isolation, and climatic stability; Körner 2000), ultimately related to speciation, extinction, colonization or co-existence. These gradients should be considered when evaluating the ecological and evolutionary mechanisms that influence species richness. For instance, if speciation is positively correlated with temperature, then negative relationships between elevation and species richness will arise because more species are produced at warmer, lower elevations; alternatively, extinction may be negatively correlated with temperature, such that high-elevation taxa go extinct more often and thus yield lower richness over time. In contrast, diversification rates may not vary with respect to climatic variables, but temperature may limit the number of species that are capable of colonizing an area to those with the appropriate traits for surviving in the cold (environmental filtering), or limit the number of species that can co-exist in a given low-temperature microhabitat (competitive exclusion) where resources are limited (Fig. 2). 


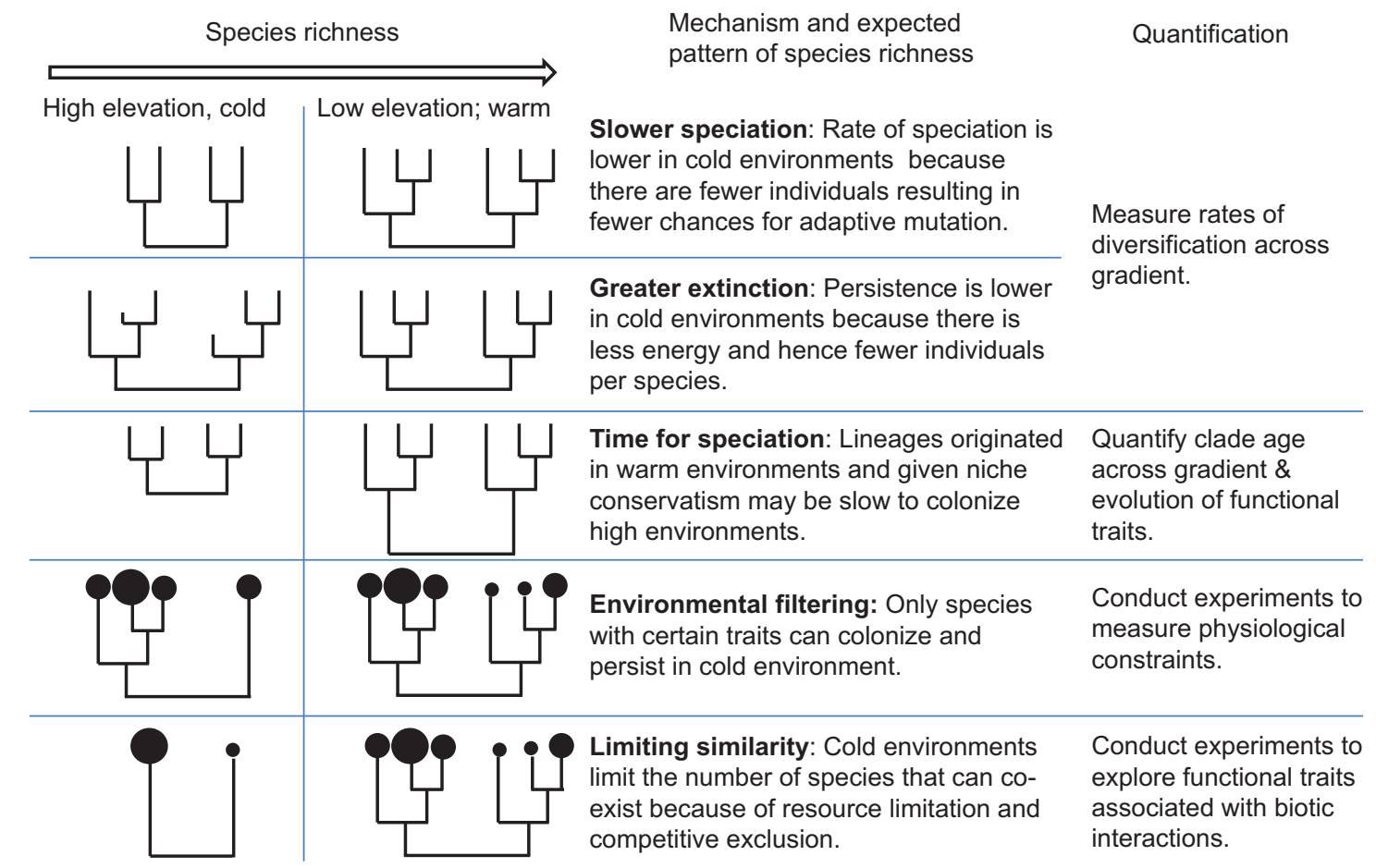

Figure 2. Example of how multiple ecological and evolutionary factors can be considered to explore patterns of richness along an elevational gradient based on the assumption that temperature is the gradient of interest.

The fundamental question is to what extent do specific ecological or evolutionary mechanisms represent plausible explanations for the generation and maintenance of patterns of diversity along elevational gradients? We describe how ecological and evolutionary approaches can, and have, been used to evaluate drivers of potential richness patterns across elevational gradients (Fig. 1, panels I and II, Fig. 2). We then describe how species-specific measurements or experiments can be used to both refine hypotheses related to the generation and maintenance of potential richness (Fig. 1, panels I and II) and hypotheses related to observed richness (Fig. 1, panels II and III, Fig. 2). The order in which research activities are presented below need not dictate how a research program should be organized, but we argue that all activities are likely illuminating.

\section{Quantify patterns of richness over the elevational gradient of interest}

Both the potential and observed pattern of richness along a given gradient (Fig. 1, panels II and III) should be quantified. For instance, what is the relationship between ant species richness and elevation within the Great Smoky Mountains National Park and for all members of that lineage across the entire Appalachian region? The simplest way to determine potential richness is to use the minimum and maximum elevational limit of species across the entire mountain region (information that is increasingly available for many taxa). Alternatively, knowledge of organismal physiology can be used to predict where each species may occur, either along the elevational gradient itself or in association with environmental variables that vary across the elevational gradient. Observed local richness should be obtained from actual field work aimed at sampling the number of locally interacting species in a community.

\section{Explore diversity-environment relationships}

Over the past several decades, a variety of empirical correlates of species richness, their form, and the potential ecological and evolutionary mechanisms proposed to underlie them, have been explored (Willig et al. 2003, Currie et al. 2004, Mittelbach et al. 2007). Consideration of these factors in relation to diversity-environment relationships will yield insight into which factors drive potential and observed richness along a given gradient (Fig. 1).

One could envision a scenario in which species richness gradients are correlated only with environmental factors, and neither reflects rates of diversification nor the time interval for which the lineage of interest has been present in a given ecological zone. If so, then ecological mechanisms, acting over short temporal and spatial scales, may have a particularly important role in generating patterns of observed species richness (Fig. $1 \mathrm{~m}$ ). In other cases, richness may be correlated with diversification rate or time for speciation (Kozak and Wiens 2012; Fig. 1h), suggesting evolutionary or historical factors, which shaped richness patterns over long time scales, may predominate. In these types of instances, the interpretation of the relative contribution of different processes may be possible. Of course, this need not be an either/or scenario, and it is difficult to imagine a scenario in which only ecological processes over short time periods or only evolutionary processes acting over longer time periods shape gradients.

In most cases, multiple factors will explain the accumulation of species over space and time (Fig. 1). Environments 
that have been inhabited the longest may harbor the most species-rich assemblages (Fjeldså 1994). Alternatively, preadaptation to some environmental conditions on the gradient may permit colonizing species to diversify in that environment (Donoghue 2008, Drummond 2008). Biotic constraints may also limit local richness; clades show diversification slowdowns where richness of a given clade is high (Wiens et al. 2011). If local assemblages fill up with species over time, then there may be no relationship between time since colonization and local richness (Rabosky 2009); instead, the latter should plateau for habitats or climatic zones that have been inhabited the longest. Such a pattern characterizes local assemblages of hylid frogs and plethodontid salamanders (Kozak and Wiens 2012), but is not consistent across many groups as indicated by the fossil record (Benton and Emerson 2007). Plotting the relationship between richness and time, estimated either from phylogenies or the fossil record, can be used to detect signatures of these processes (Benton and Emerson 2007, Rabosky 2009, Rabosky and Glor 2010).

\section{Evaluate patterns of phylogenetic or trait assemblage structure}

Patterns of phylogenetic or trait structure can provide some insights into the potential importance of environmental filtering or biotic interactions (notably competition) in structuring local assemblages (Webb et al. 2002). If environmental filtering is the predominant mechanism structuring a local assemblage and functional traits are conserved on the phylogeny then closely related species with similar traits are predicted to co-occur. In contrast, biotic interactions, including competition or enemy-mediated negative diversity dependence, should result in co-occurrence of unrelated species with even spacing of traits values. While this framework can provide useful insights about factors influencing assemblage structure (Emerson and Gillespie 2008, Cavender-Bares et al. 2009), it also has several weaknesses (Mayfield and Levine 2010, Losos 2011, Aiba et al. 2013), some of which can be overcome with targeted experiments or measurements.

\section{Conduct targeted experiments or measurements}

Experiments can serve two roles: to evaluate the insights gained using ecological or phylogenetically-based correlative approaches aimed at understanding potential richness (Fig. 1, panels I and II), and to explore the existence and pattern of biotic interactions (Fig. 1, panels II and III). Direct physiological measurements or a combination of greenhouse, common-garden, and reciprocal-transplant experiments can be used to identify the environmental tolerances of species with different traits and/or relatedness patterns (Jankowski et al. 2013). Confirmation of the potential importance of such traits, combined with knowledge of how these traits evolved, is critical if we are to understand the mechanistic underpinnings of richness-environment relationships from macroecological, phylogenetically-based or trait-based hypotheses. Although more difficult to implement, direct experimental manipulations of the abiotic environments along elevational gradients could provide further insight into the role of environmental constraints on patterns of potential richness. For example, hydric supplementation and addition of materials that increase thermal conductance may be applied to create wetter or warmer local environments, or shade tables can be used to lower temperatures (Lessard et al. 2011). If richness changes with manipulation of the local environment, then environmental constraints may play an important role in controlling species numbers in local assemblages, at least on short time scales (Cavieres et al. 2014). Long-term experiments allowing for new colonists to arrive after manipulation would provide more complete insights into the influence of the manipulation on local species richness.

Experimental methods can also be used to assess the role of biotic factors in shaping patterns of diversity (Fig. 1, panels II and III). Biotic mechanisms can be assessed in experiments where related taxa or species with similar functional traits are placed together (e.g. plant plots or mesocosms) and monitored through time. For instance, removal experiments at low and high elevations suggested important roles for both positive and negative interactions in affecting plant fitness and ultimately species co-occurrence in montane systems (Callaway et al. 2002). While such experiments will be limited to certain types of organisms and have been most often conducted on plants, some animal systems can also be manipulated. For instance, Gifford and Kozak (2012) combined physiological measurements and experiments of competitive abilities to explore the role of competition shaping altitudinal diversity gradients in salamanders. Knowledge of which traits confer changes in fitness in a given environment (and how those traits are distributed on a phylogeny) provide additional insight into which traits are most important in abiotic (e.g. environmental tolerance) and biotic (e.g. competitive interactions, resistance to herbivores or pathogens) interactions, promoting insight about how these traits may be evolutionarily constrained (Savage and Cavender-Bares 2012).

Manipulative experiments are not viable in every system and for every taxon, however, when appropriate, experiments provide compelling evidence. When they are not appropriate (e.g. we are not suggesting moving Sequoiadendron giganteum trees up and down the elevational gradient in the Sierra Mountains), other approaches, such as the community phylogenetics approach can provide some insights. However, our suggestion would be to not rely solely on phylogenetic patterns to infer underlying processes (Mayfield and Levine 2010, Losos 2011).

\section{Challenges and perspectives}

True integration between ecological and evolutionary determinants of species richness gradients on mountains, and most other systems, has yet to be achieved. This is due in part to limitations of analytical and experimental methods, lack of fine-scale richness and environmental data, and lack of communication among fields. However, the continued interest in diversity patterns is forging links among disciplines, resulting in new hypothesis frameworks (Weber and Agrawal 2012) and data sources (Kattge et al. 2011, Brewer et al. 2012). Nonetheless, three main challenges remain. The first is driven by the temporal limitations of available data (fine-scale environmental data on evolutionary time scales 
are not available) and inference tools, the second is related to lack of knowledge of traits that allow species to inhabit certain portions of the gradient, and the third stems from a need to compare multiple transects within a montane region as well as multiple montane regions with different characteristics in order to evaluate the generality of different drivers of diversity. While significant, these challenges can be overcome with on-going refinement of historical and geological data and an increased research emphasis on studying and conserving montane biodiversity.

Patterns of species richness could depend on the deeper geographic history of a region and on the fluctuation in environmental conditions at the time that montane systems were forming (Päckert et al. 2012). For instance, species-environment relationships that consider how climate and productivity varied through geological time suggest that time-integrated measures of these variables may be better predictors of current species richness than contemporary measures (Jetz and Fine 2012). In addition, high environmental instability may promote extinction, whereas stability allows the build-up of lineages through time, resulting in higher species richness and endemism in more stable areas (Graham et al. 2006). Phylogeographic studies in several regions confirm that areas where lineages persisted over time were historically stable (Carnaval et al. 2009, Velo-Antón et al. 2013). While these examples illustrate the importance of considering how climate and geological history influence current patterns of diversity, such endeavors are still scarce and limited by the precision and availability of historical data. Attempts to directly link diversification history with historical data are hampered by uncertainty in the age of nodes on phylogenetic hypotheses, by the coarse spatial and temporal scale of most historical climatic and geological data, and by the difficulties of separately estimating speciation and extinction rates from phylogenetic hypotheses based on current taxa (Quental and Marshall 2010). Continued research efforts in these areas should provide new data and tools to more rigorously address some aspects of our research framework.

We know surprisingly little about specific traits or physiological adaptations that allow species to inhabit certain portions of the gradient and how these traits evolve. For instance, species that occur at high elevations often have speciesspecific physiological mechanisms that permit them to persist under cold, low-oxygen conditions; these mechanisms are unknown for most species (Navas and Chaui-Berlinck 2007). Increased species-specific knowledge will aid in several steps in our framework including quantifying the potential species pool using context-appropriate physiological data, or identifying environmental gradients appropriate for investigation and experimentation.

The final challenge of our framework stems from the fact that generalization about the importance of different mechanisms influencing current patterns of diversity requires multiple transects within a montane region and multiple montane regions with different environmental attributes. Experiments at this scale may not be feasible with many organisms and by a single research team. In addition, experiments can measure transient dynamics and researchers should guard against over-interpreting the results of experiments or short-term observations (Wiens 1989). Nonetheless, generalizations should emerge from replication of observational and experimental studies in different contexts (Wang et al. 2009). Efforts aimed at sharing data among investigators and across organisms with varied traits will enhance our ability to search for generality of patterns among montane systems. As our research becomes comparative and expands to include different mountain systems and experimental manipulations, we predict that this integrative framework will identify general mechanisms underlying patterns of montane diversity.

Acknowledgements - This study was conducted as part of the working group Montane Biodiversity in Space and Time: Linking Evolutionary Biology and Macroecology, supported by the National Evolutionary Synthesis Center (NESCent), NSF EF-0423461. We thank Adam Algar, Jordi Bascompte, Jonathon Chase, Marcel Holyoak, and Walter Jetz for helpful comments on earlier versions of the manuscript.

Author contribution: the ideas for this paper were generated at a NESCent working group entitled: 'The origin and maintenance of montane biodiversity: integrating evolutionary and ecological processes', organized by $\mathrm{CHG}, \mathrm{KHK}$ and $\mathrm{CR}$. CHG primarily wrote the manuscript; NJS, ACA, CDC, TER, KRZ, KHK and $\mathrm{CR}$ contributed to manuscript preparation and writing; all authors read and commented on the manuscript.

\section{References}

Adams, D. C. and Rohlf, F. J. 2000. Ecological character displacement in Plethodon: biomechanical differences found from a geometric morphometric study. - Proc. Natl Acad. Sci. USA 97: 4106-4111.

Aiba, M. et al. 2013. Robustness of trait distribution metrics for community assembly studies under the uncertainties of assembly processes. - Ecology 94: 2873-2885.

Algar, A. C. et al. 2011. Quantifying the importance of regional and local filters for community trait structure in tropical and temperate zones. - Ecology 92: 903-914.

Benton, M. J. and Emerson, B. C. 2007. How did life become so diverse? The dynamics of diversification according to the fossil record and molecular phylogenetics. - Palaeontology 50: 23-40.

Bertrand, R. et al. 2011. Changes in plant community composition lag behind climate warming in lowland forests. - Nature 479: 517-520.

Brewer, S. et al. 2012. Paleoecoinformatics: applying geohistorical data to ecological questions. - Trends Ecol. Evol. 27: $104-112$.

Callaway, R. M. et al. 2002. Positive interactions among alpine plants increase with stress. - Nature 417: 844-848.

Carnaval, A. C. et al. 2009. Stability predicts genetic diversity in the Brazilian Atlantic Forest hotspot. - Science 323: 785-789.

Carstensen, D. W. et al. 2013. Introducing the biogeographic species pool. - Ecography 36: 1310-1318.

Cavender-Bares, J. et al. 2004. Phylogenetic overdispersion in Floridian oak communities. - Am. Nat. 163: 823-843.

Cavender-Bares, J. et al. 2009. The merging of community ecology and phylogenetic biology. - Ecol. Lett. 12: 693-715.

Cavieres, L. A. et al. 2014. Facilitative plant interactions and climate simultaneously drive alpine plant diversity. - Ecol. Lett. 17: 193-202.

Currie, D. J. et al. 2004. Predictions and tests of climate-based hypotheses of broad-scale variation in taxonomic richness. - Ecol. Lett. 7: 1121-1134. 
Davies, T. J. et al. 2007. Species co-existence and character divergence across carnivores. - Ecol. Lett. 10: 146-152.

Diamond, S. E. et al. 2012. Who likes it hot? A global analysis of the climatic, ecological, and evolutionary determinants of warming tolerance in ants. - Global Change Biol. 18: 448-456.

Donoghue, M. J. 2008. A phylogenetic perspective on the distribution of plant diversity. - Proc. Natl Acad. Sci. USA 105: 11549-11555.

Drummond, C. S. 2008. Diversification of Lupinus (Leguminosae) in the western New World: derived evolution of perennial life history and colonization of montane habitats. - Mol. Phylogenet. Evol. 48: 408-421.

Emerson, B. C. and Gillespie, R. G. 2008. Phylogenetic analysis of community assembly and structure over space and time. - Trends Ecol. Evol. 23: 619-630.

Fierer, N. et al. 2011. Microbes do not follow the elevational diversity patterns of plants and animals. - Ecology 92: 797-804.

Fjeldså, J. 1994. Geographical patterns for relict and young species of birds in African and South America and implications for conservation priorities. - Biodivers. Conserv. 3: 207-226.

Gifford, M. E. and Kozak, K. H. 2012. Islands in the sky or squeezed at the top? Ecological causes of elevational range limits in montane salamanders. - Ecography 35: 193-203.

Gotelli, N. J. 2000. Null model analysis of species co-occurrence patterns. - Ecology 81: 2606-2621.

Graham, C. H. et al. 2006. Habitat history improves prediction of biodiversity in rainforest fauna. - Proc. Natl Acad. Sci. USA 103: 632-636.

Graves, G. R. 1985. Elevational correlates of speciation and intraspecific geographic variation in plumage in Andean forest birds. - Auk 102: 556-579.

Harrison, S. and Grace, J. B. 2007. Biogeographic affinity helps explain productivity-richness relationships at regional and local scales. - Am. Nat. 170: S5-S15.

Harrison, S. and Cornell, H. 2008. Toward a better understanding of the regional causes of local community richness. - Ecol. Lett. 11: 969-979.

Hawkins, B. A. et al. 2005. Water links the historical and contemporary components of the Australian bird diversity gradient. - J. Biogeogr. 32: 1035-1042.

Hendry, A. P. and Kinnison, M. T. 1999. Perspective: the pace of modern life: measuring rates of contemporary microevolution. - Evolution 53: 1637-1653.

Jankowski, J. E. et al. 2013. Exploring the role of physiology and biotic interactions in determining elevational ranges of tropical animals. - Ecography 36: doi: 10.1111/j.1600-0587. 2013.00600.x

Jetz, W. and Fine, P. V. A. 2012. Global gradients in vertebrate diversity predicted by historical area-productivity dynamics and contemporary environment. - PLoS Biol. 10: e1001292.

Jetz, W. et al. 2012. Integrating biodiversity distribution knowledge: toward a global map of life. - Trends Ecol. Evol. 27: 151-159.

Kattan, G. H. and Franco, P. 2004. Bird diversity along elevational gradients in the Andes of Colombia: area and mass effects. - Global Ecol. Biogeogr. 13: 451-458.

Kattge, J. et al. 2011. TRY - a global database of plant traits. - Global Change Biol. 17: 2905-2935.

Kisel, Y. and Barraclough, T. G. 2010. Speciation has a spatial scale that depends on levels of gene flow. - Am. Nat. 175: 316-334.

Körner, C. 2000. Why are there global gradients in species richness? Mountains might hold the answer. - Trends Ecol. Evol. 15: 513-514.

Körner, C. 2007. The use of 'altitude' in ecological research. - Trends Ecol. Evol. 22: 569-574.
Kozak, K. H. and Wiens, J. J. 2010. Accelerated rates of climaticniche evolution underlie rapid species diversification. - Ecol. Lett. 13: 1378-1389.

Kozak, K. H. and Wiens, J. J. 2012. Phylogeny, ecology, and the origins of climate-richness relationships. - Ecology 93: S167-S182.

La Sorte, F. A. and Jetz, W. 2010. Projected range contractions of montane biodiversity under global warming. - Proc. R. Soc. B 277: 3401-3410.

Lessard, J. P. et al. 2011. Determinants of the detrital arthropod community structure: the effects of temperature and resources along an environmental gradient. - Oikos 120: 333-343.

Lessard, J. P. et al. 2012. Strong influence of regional species pools on continent-wide structuring of local communities. - Proc. R. Soc. B 279: 266-274.

Li, J. et al. 2009. Climate and history explain the species richness peak at mid-elevation for Schizothorax fishes (Cypriniformes: Cyprinidae) distributed in the Tibetan Plateau and its adjacent regions. - Global Ecol. Biogeogr. 18: 264-272.

Loarie, S. R. et al. 2009. The velocity of climate change. - Nature 462: 1052-1055.

Losos, J. B. 2011. Seeing the forest for the trees: the limitations of phylogenies in comparative biology. - Am. Nat. 177: 709-727.

Losos, J. B. and Schluter, D. 2000. Analysis of an evolutionary species-area relationship. - Nature 408: 847-850.

MacArthur, R. H. 1972. Geographical ecology: patterns in the distribution of species. - Harper and Row.

MacArthur, R. and Levins, R. 1967. Limiting similarity convergence and divergence of coexisting species. - Am. Nat. 101: 377-385.

Machac, A. et al. 2013. Ecological causes of decelerating diversification in Carnivoran mammals - Evolution 67: 2423-2433.

Mayfield, M. M. and Levine, J. M. 2010. Opposing effects of competitive exclusion on the phylogenetic structure of communities. - Ecol. Lett. 13: 1085-1093.

McCain, C. M. and Grytnes, J. A. 2010. Elevational gradients in species richness. - In: Encyclopedia of Life. Wiley.

McCain, C. M. and Colwell, R. K. 2011. Assessing the threat to montane biodiversity from discordant shifts in temperature and precipitation in a changing climate. - Ecol. Lett. 14: 1236-1245.

Mittelbach, G. G. et al. 2007. Evolution and the latitudinal diversity gradient: speciation, extinction and biogeography. - Ecol. Lett. 10: 315-331.

Navas, C. A. and Chaui-Berlinck, J. G. 2007. Respiratory physiology of high-altitude anurans: 55 years of research on altitude and oxygen. - Respir. Physiol. Neurol. 158: 307-313.

Päckert, M. et al. 2012. Horizontal and elevational phylogeographic patterns of Himalayan and southeast Asian forest passerines (Aves: Passeriformes). - J. Biogeogr. 39: 556-573.

Quental, T. B. and Marshall, C. R. 2010. Diversity dynamics: molecular phylogenies need the fossil record. - Trends Ecol. Evol. 25: 434-441.

Rabosky, D. L. 2009. Ecological limits and diversification rate: alternative paradigms to explain the variation in species richness among clades and regions. - Ecol. Lett. 12: 735-743.

Rabosky, D. L. and Glor, R. E. 2010. Equilibrium speciation dynamics in a model adaptive radiation of island lizards. - Proc. Natl Acad. Sci. USA 107: 22178-22183.

Rahbek, C. 1995. The elevational gradient of species richness - a uniform pattern. - Ecography 18: 200-205.

Rahbek, C. 1997. The relationship among area, elevation, and regional species richness in neotropical birds. - Am. Nat. 149: 875-902. 
Rahbek, C. 2005. The role of spatial scale and the perception of large-scale species-richness patterns. - Ecol. Lett. 8: 224-239.

Rahbek, C. and Graves, G. R. 2001. Multiscale assessment of patterns of avian species richness. - Proc. Natl Acad. Sci. USA 98: 4534-4539.

Ricklefs, R. E. 2004. A comprehensive framework for global patterns in biodiversity. - Ecol. Lett. 7: 1-15.

Ricklefs, R. E. 2006. Global variation in the diversification rate of passerine birds. - Ecology 87: 2468-2478.

Sanders, N. J. et al. 2003. Patterns of ant species richness along elevational gradients in an arid ecosystem. - Global Ecol. Biogeogr. 12: 93-102.

Savage, J. A. and Cavender-Bares, J. 2012. Habitat specialization and the role of trait lability in structuring diverse willow (genus Salix) communities. - Ecology 93: S138-S150.

Schemske, D. W. et al. 2009. Is there a latitudinal gradient in the importance of biotic interactions? - Annu. Rev. Ecol. Evol. Syst. 40: 245-269.

Schluter, D. and McPhail, J. D. 1992. Ecological character displacement and speciation in sticklebacks. - Am. Nat. 140: 85-108.

Stevens, R. D. 2006. Historical processes enhance patterns of diversity along latitudinal gradients. - Proc. R. Soc. B 273: 2283-2289.

Stevens, R. D. 2011. Relative effects of time for speciation and tropical niche conservatism on the latitudinal diversity gradient of phyllostomid bats. - Proc. R. Soc. B 278: 2528-2536.

Sundqvist, M. K. et al. 2013. Community and ecosystem responses to elevational gradients: processes, mechanisms, and insights for global change. - Annu. Rev. Ecol. Evol. Syst. 44: 261-280.

Terborgh, J. W. and Faaborg, J. 1980. Saturation of bird communities in the West-Indies. - Am. Nat. 116: 178-195.

Velo-Anton, G. et al. 2013. Tracking climate change in a dispersallimited species: reduced spatial and genetic connectivity in a montane salamander. - Mol. Ecol. 22: 3261-3278.

Wang, X. P. et al. 2009. Relative importance of climate vs local factors in shaping the regional patterns of forest plant richness across northeast China. - Ecography 32: 133-142.

Webb, C. O. et al. 2002. Phylogenies and community ecology. Annu. Rev. Ecol. Syst. 33: 475-505.

Weber, M. G. and Agrawal, A. A. 2012. Phylogeny, ecology, and the coupling of comparative and experimental approaches. Trends Ecol. Evol. 27: 394-403.

Werenkraut, V. and Ruggiero, A. 2011. Quality of basic data and method to identify shape affect richness-altitude relationships in meta-analysis. - Ecology 92: 253-260.

Wiens, J. A. 1989. Spacial scaling in ecology. - Funct. Ecol. 3: 385-397.

Wiens, J. J. et al. 2007. Phylogenetic history underlies elevational biodiversity patterns in tropical salamanders. - Proc. R. Soc. B 274: 919-928.

Wiens, J. J. et al. 2011. Phylogenetic origins of local-scale diversity patterns and the causes of Amazonian megadiversity. - Ecol. Lett. 14: 643-652.

Willig, M. R. et al. 2003. Latitudinal gradients of biodiversity: pattern, process, scale, and synthesis. - Annu. Rev. Ecol. Evol. Syst. 34: 273-309. 